\title{
Discrete Lorentz symmetry and discrete spacetime translational symmetry in two- and three-dimensional crystals
}

\author{
Xiuwen Li, ${ }^{1}$ Jiaxue Chai, ${ }^{1}$ Huixian Zhu, ${ }^{1}$ and Pei Wang ${ }^{1, *}$ \\ ${ }^{1}$ Department of Physics, Zhejiang Normal University, Jinhua 321004, China
}

(Dated: March 30, 2020)

\begin{abstract}
As is well known, crystals have discrete space translational symmetry. It was recently noticed that one-dimensional crystals possibly have discrete Poincaré symmetry, which contains discrete Lorentz and discrete time translational symmetry as well. In this paper, we classify the discrete Poincaré groups on two- and three- dimensional Bravais lattices. They are the candidate symmetry groups of two- or three-dimensional crystals, respectively. The group is determined by an integer generator $g$, and it reduces to the space group of crystals at $g=2$.
\end{abstract}

\section{INTRODUCTION}

After the papers by Wilczek and Shapere ${ }^{1,2}$, the spontaneous breaking of time translational symmetry (TTS) becomes a focus of research and controversy ${ }^{3}-\underline{-9}$. According to the special theory of relativity, time and space must be put on the same footing. The existence of crystals is a manifestation of space translational symmetry (STS) being spontaneously broken into a discrete one ${ }^{10}$. It is then natural to think that TTS can also be spontaneously broken into a discrete one.

It was soon noticed that the original definition of TTS breaking by Wilczek is problematic. Bruno ${ }^{3}$, and Watanabe and Oshikawa ${ }^{4}$, proved that continuous TTS cannot be broken in the ground state or Gibbs ensemble of a quantum system. But this does not rule out the possibility of TTS breaking in nonequilibrium states. The spontaneous breaking of TTS was redefined for periodically driven systems ${ }^{5}$. The proposals for experiments were discussed ${ }^{6.7}$ and realized in $2016^{8.9}$.

In the theory of special relativity, STS and TTS are connected to each other by a rotation in spacetime, i.e. the Lorentz transformation. Lagrangian must be invariant under both the Lorentz transformations and spacetime translations, which combine into the Poincaré group 11. The low-energy states may have less symmetries. After a process called spontaneous symmetry breaking, the symmetry group reduces to a subgroup of the Poincaré group. Examples are the space groups of crystals. It was long believed that crystals have discrete STS, continuous TTS, but no Lorentz symmetry.

An exceptional possibility was discussed in Ref. [12]. In $1+1$ dimensions, the Poincaré group has subgroups that include both discrete spacetime translations and discrete Lorentz transformations. A crystal cannot have continuous Lorentz symmetry, but it can have discrete one. As thus, it must have discrete TTS as well. The overall translational symmetry is determined by a lattice in $1+1$ dimensions. And the period of TTS is connected to the lattice constant of crystal and the speed of light. The possibility of crystals owning discrete TTS and Lorentz symmetry has not been noticed before. Indeed, no observation of such symmetry was reported up to now. A possible explanation is that the period of TTS is too small, only in the order of $10^{-18} s$.

In this paper, we generalize above results to higher dimensions. We classify the discrete Poincaré groups in $1+2$ and $1+3$ dimensions. They are the candidate symmetry groups of the corresponding two- and threedimensional crystals, respectively. The STS of a crystal is given by its Bravais lattice. There are 5 and 14 Bravais lattices in two and three spatial dimensions, respectively. We find discrete Poincaré symmetry only on six of them. Table【enumerates these lattices and the discrete symmetry on them. Our results have potential application in the search of exotic symmetry of crystals. Our finding might also be interesting to ones who study quantum gravity. Some approaches to quantum gravity propose the spacetime to have a lattice structure ${ }^{13-18}$. And the study on discrete Poincaré groups provides a way of maintaining the Lorentz symmetry on a spacetime lattice.

The paper is organized as follows. In Sec. II we review the discrete Poincaré group in $1+1$ dimensions, and then introduce the method of generalization to higher dimensions. Sec. III and IV contribute to $1+2$ and $1+3$ dimensions, respectively. Sec. $\mathrm{V}$ discusses the possible way of observing discrete Poincaré symmetry in crystals.

\section{DISCRETE POINCARÉ SYMMETRY}

Crystals have discrete STS. Their local properties change periodically in space. In $1+1$ dimensions, a crystal looks the same under a spatial translation of coordinates if and only if the translation distance is an integer times of the lattice constant. Between two reference frames that are moving relative to each other, the coordinates transform as a Lorentz transformation. Usually, crystals look different in different reference frames. But Ref. 12] showed that it is possible for crystals to look the same if the relative velocity takes some specific values. The cost is that the properties of crystals must change with time at some specific periods. This kind of symmetry is called the discrete Poincaré symmetry.

Ref. 12 discussed in general how to construct a periodic function of spacetime that is invariant under a group of Lorentz transformations. STS of a crystal is determined by its Bravais lattice. The 1D Bravais lattice 


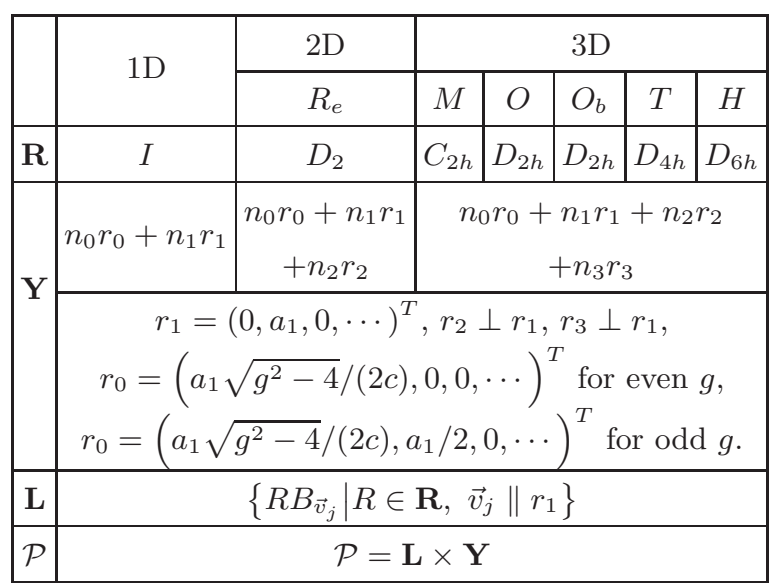

TABLE I. Discrete Poincaré groups (denoted by $\mathcal{P}$ ) on the one-dimensional (1D), two-dimensional (2D) and threedimensional (3D) Bravais lattices. $R_{e}$ denotes the rectangular lattice. $M, O, O_{b}, T$ and $H$ denote the monoclinic, orthorhombic, base-centered orthorhombic, tetragonal and hexagonal lattices, respectively. $\mathbf{R}$ is the point group of the corresponding Bravais lattice. The set $\mathcal{P}$ is a direct product of $\mathbf{L}$ and $\mathbf{Y}$, which are the groups of Lorentz transformations and spacetime translations, respectively. The translation group $\mathbf{Y}$ forms a spacetime lattice. Each vector of $\mathbf{Y}$ is a linear combination of its primitive vectors. $n_{0}, n_{1}, n_{2}$ and $n_{3}$ are integers. $r_{1}, r_{2}$ or $r_{3}$ are the primitive vectors of the corresponding Bravais lattice, while $r_{0}$ is the temporal primitive vector. In the expression of $r_{0}$ and $r_{1}, a_{1}$ denotes the lattice constant, $c$ denotes the speed of light, and $g \geq 2$ is an integer generator which determines the shape of $\overline{\mathbf{Y}}$. The discrete Lorentz group $\mathbf{L}$ contains the elements of $\mathbf{R}$, as well as the Lorentz boosts $B_{\vec{v}_{j}}$. The velocity $\vec{v}_{j}$ in the Lorentz boost must be in the direction of $r_{1}$, and can only take some discrete values $(j$ is an integer).

can be extended in the time direction to form a $1+1$ dimensional spacetime lattice. For simplicity, let us view a crystal as a spacetime lattice. Under specific Lorentz transformations, this lattice keeps the same in spite of length contraction and time dilation. This is possible if the spatial direction after transformation is still in one of the lattice directions. The spatial directions vary from one reference frame to the other, but the lattice constant, i.e. the distance between two neighbor sites in the spatial direction, keeps the same. Notice that two spatial neighbor sites in one reference frame are not spatial neighbors in the other because they are not simultaneous any more.

All the coordinate transformations that keep a crystal invariant make up a group, dubbed the discrete Poincaré group. It contains the discrete Lorentz transformations and the spacetime translation of lattice vectors. The element of a discrete Poincaré group is denoted by $\Lambda(L, r)$, which means a Lorentz transformation $L$ followed by a spacetime translation $r$. In $1+1$ dimensions, $L$ is a 2 -by- 2 matrix and $r$ is a two-components vector. According to definition, the product of two group elements reads

$$
\Lambda\left(L^{\prime}, r^{\prime}\right) \Lambda(L, r)=\Lambda\left(L^{\prime} L, L^{\prime} r+r^{\prime}\right) .
$$

The discrete Poincaré group is found to be

$$
\mathcal{P}=\left\{\Lambda\left(L_{v_{j}}, r_{n_{0} n_{1}}\right) \mid j, n_{0}, n_{1}=0, \pm 1, \pm 2, \cdots\right\},
$$

where $j, n_{0}$ and $n_{1}$ are arbitrary integers. In the Lorentz transformation, $v_{j}$ denotes the velocity of one reference frame relative to the other, taking the value $v_{j} / c=$ $\operatorname{sign}(j) \sqrt{1-4 / m_{j}^{2}}$ with $c$ the speed of light. And $m_{j}$ is an integer sequence satisfying $m_{j+1}=g m_{j}-m_{j-1}$, where $g$ is the generator of the sequence, $m_{0}=2, m_{1}=g$ and $m_{-j}=m_{j}$. The translation vector $r_{n_{0} n_{1}}$ reads

$$
r_{n_{0} n_{1}}=n_{0} r_{0}+n_{1} r_{1}
$$

where $r_{1}=\left(0, a_{1}\right)^{T}$ with $a_{1}$ denoting the lattice constant. And $r_{0}$ equals $\left(a_{1} \sqrt{g^{2}-4} /(2 c), 0\right)^{T}$ for even $g$ or $\left(a_{1} \sqrt{g^{2}-4} /(2 c), a_{1} / 2\right)^{T}$ for odd $g$. Note that the first component of a vector denotes the time coordinate, while the second denotes the space coordinate.

A discrete Poincaré group is determined by its generator $g(g \geq 2)$. As $g=2$, we have $m_{j} \equiv 2$ and $v_{j} \equiv 0$. $\mathcal{P}$ contains no Lorentz transformation, and the translation vector $r_{n_{0} n_{1}}$ is purely spatial. This is the symmetry group of $1 \mathrm{D}$ crystals in the orthodox view.

In the case $g>2$, the spatial translations $\left(n_{0}=0\right)$ in $\mathcal{P}$ keep the same. But $\mathcal{P}$ contains additional discrete Lorentz transformations and discrete TTS. As $g>2$ is even, the points $r_{n_{0} n_{1}}$ form a rectangular lattice in $1+1$ dimensional spacetime. This lattice gives the spacetime translational symmetry. Both TTS and STS are discrete, which are connected to each other by the discrete Lorentz rotations $L_{v_{j}}$. The period of TTS is $\sqrt{g^{2}-4} a /(2 c)$. As $g$ is odd, the points $r_{n_{0} n_{1}}$ form a centered rectangular lattice. The period of TTS becomes $\sqrt{g^{2}-4} a / c$. The velocity $v_{j}$ in the Lorentz rotation can only take discrete values. For example, $v_{j}$ takes $0, \sqrt{5} c / 3,3 \sqrt{5} c / 7, \cdots$ at $g=3$, or $0, \sqrt{3} c / 2,4 \sqrt{3} c / 7, \cdots$ at $g=4$.

$\mathcal{P}$ is the subgroup of the continuous Poincaré group for whatever $g$. It is reasonable to guess that $\mathcal{P}$ at $g>2$ is also the symmetry group of some crystals. If a crystal chooses $\mathcal{P}$ at $g>2$ as its symmetry group, it looks the same after a coordinate translation only if the translation vector is $r_{n_{0} n_{1}}$. It means that the local properties are varying not only with space but also with time. They are periodic functions of spacetime coordinates.

A few more words are necessary for explaining the physical meaning of $v_{j}$. Let us view a crystal as a chain of atoms with the distance between two neighbors being a. The discrete TTS requires that the atoms are moving periodically even in the rest frame (like a lattice vibration). If all the atoms are oscillating in the same phase, $a$ becomes the period in the spatial direction (the lattice constant). But in a moving reference frame, the atoms are not oscillating in the same phase anymore, because simultaneity depends on the reference frame. Starting 
from an atom $A$, we will find that the movement of its neighbor is now behind $A$. But since the oscillation is periodic, after a few atoms, we may again find an atom $B$ that is oscillating in the same phase as $A$. Now the lattice constant becomes the distance between $A$ and $B$. But due to the length contraction, this distance is indeed $a$ in the moving reference frame. For this to happen, the contraction must be strong, since the distance between $A$ and $B$ in the rest frame is a few times of $a$. This explains why $v_{j}$ is comparable to the speed of light.

Eq. (2) gives the discrete Poincaré groups in $1+1$ dimensions. In this paper, we generalize to $1+2$ and $1+3$ dimensions. For this purpose, we define the symmorphic Poincaré group. The element $\Lambda(L, r)$ is a pure Lorentz transformation as $r=0$, or a pure translation as $L=1$ (the identity matrix). We use $\mathbf{L}=\{L\}$ to denote a group of Lorentz transformations, and $\mathbf{Y}=\{r\}$ to denote a group of translations. If the set $\mathcal{P}$ is a direct product of $\mathbf{L}$ and $\mathbf{Y}$ and $\mathcal{P}$ is a group under the multiplication rule (11), we say that $\mathcal{P}$ is a symmorphic Poincaré group. This definition is similar to the symmorphic space group in crystallography. In $1+1$ dimensions, the discrete Poincaré group is indeed a symmorphic group.

In $d \geq 2$ spatial dimensions, the Lorentz transformation $L \in \mathbf{L}$ is a $(1+\mathrm{d})$-by- $(1+\mathrm{d})$ matrix. The translation $r \in \mathbf{Y}$ is a $(1+d)$-dimensional vector. $L$ acting on $r$ gives the transformation of a vector between different reference frames. If $L r$ and $L^{-1} r$ are both the elements of $\mathbf{Y}$ for each $r \in \mathbf{Y}$, we say that $\mathbf{Y}$ is invariant under $L$. Note that $\mathbf{Y}$ is indeed a lattice in $(1+d)$-dimensional spacetime. $\mathbf{Y}$ being invariant under $L$ means that this lattice keeps the same after the spacetime rotation $L$. If $\mathbf{Y}$ is invariant under each $L \in \mathbf{L}$, we say that $\mathbf{Y}$ is invariant under L. We construct discrete Poincaré groups based on next fact: $\mathbf{L} \times \mathbf{Y}$ is a symmorphic Poincaré group if and only if $\mathbf{Y}$ is invariant under $\mathbf{L}$. The proof is given in appendix $\mathrm{A}$

In $d \geq 2$ spatial dimensions, symmetry operations include rotation, reflection, inversion and improper reflection. These operations are denoted by $R$. And we use $B$ to denote a Lorentz boost (a symmetric matrix in the unit $c=1$ ). In general, a Lorentz transformation in $1+\mathrm{d}$ dimensions can be expressed as $L=R B$. In $d \geq 2$ spatial dimensions, the group $\mathbf{L}$ contains not only Lorentz boosts, but also spatial rotations, reflections, etc.. Indeed, it is not enough to identify a reference frame by just giving its velocity. Because different reference frames may differ by a rotation. This complexity causes all the difficulties in the construction of discrete Poincaré groups.

In our approach, we start from a d-dimensional Bravais lattice, extending it in the time direction to form a $(1+d)$ dimensional lattice $\mathbf{Y}$. Therefore, $\mathbf{Y}$ contains the Bravais lattice as its part. This is what we require for $\mathcal{P}=\mathbf{L} \times \mathbf{Y}$ being the symmetry group of a Bravias lattice.

We then check if $\mathbf{Y}$ is invariant under the Lorentz boosts, rotations, reflections, etc.. These operations make up the group $\mathbf{L}$. L contains the point group of the Bravais lattice. For example, for the 2D square lattice, $\mathbf{L}$ must contain the spatial rotations of angles 0 , $\pi / 2, \pi$ or $3 \pi / 2$. If $\mathbf{L}$ contains nothing more than the point group, $\mathbf{Y}$ is obviously invariant under $\mathbf{L}$ (a Bravais lattice is invariant under its point group according to definition). In this case, we obtain a trivial symmorphic Poincaré group with no Lorentz transformation. It is the symmetry group of crystals in the orthodox view.

For $\mathcal{P}$ to be nontrivial, $\mathbf{L}$ must contain at least one Lorentz boost $B_{\vec{v}}$ with $\vec{v} \neq 0^{19}$. We find that $\mathbf{Y}$ is invariant under $B_{\vec{v}} \in \mathbf{L}$ only if $\vec{v}$ is in the lattice direction, that is $\vec{v}$ connects at least two sites of the Bravais lattice (see appendix B for the proof). Without loss of generality, we suppose that $\vec{v}$ is in the $x$-direction. The sites of $\mathbf{Y}$ in the $x$-axis then form a 1D Bravais lattice. Eq. (3) has told us the unique way of extending a 1D Bravais lattice into a $(1+1)$-dimensional spacetime lattice. In this way, we naturally obtain the sublattice of $\mathbf{Y}$ in the $t$ - $x$ plane. We can then obtain the whole $\mathbf{Y}$ and $\mathcal{P}=\mathbf{L} \times \mathbf{Y}$ (see the detailed derivation in appendix $[\mathrm{C}$ and $\mathrm{D}) . \mathcal{P}$ is the subgroup of the continuous Poincaré group, at the same time contains the space group of Bravais lattice as its subgroup. In next sections, we enumerate $\mathcal{P}$ in $1+2$ and $1+3$ dimensions.

\section{TWO DIMENSIONAL BRAVAIS LATTICES}

In two spatial dimensions, there are five Bravais lattices: oblique, rectangular, centered rectangular, hexagonal and square lattices. There exist symmorphic Poincaré groups on the rectangular lattice, but no symmorphic Poincaré groups on the other four lattices (see table \). The derivation is given in appendix C.

Recall that an element of the symmorphic Poincaré group is denoted as $\Lambda(L, r)$, where $L$ is an element of $\mathbf{L}$ and $r$ is an element of $\mathbf{Y}$. Here $\mathbf{L}$ is the group of Lorentz transformations and $\mathbf{Y}$ is the group of translations. $r$ is a vector in 1+2-dimensional spacetime, and can be generally expressed as $r=(t, x, y)^{T}$ where $t$ denotes the time and $x$ and $y$ denote the space coordinates. Two primitive vectors of the rectangular lattice are $r_{1}=\left(0, a_{1}, 0\right)^{T}$ and $r_{2}=\left(0,0, a_{2}\right)^{T}$ with $a_{1} \neq a_{2}$.

The symmorphic Poincaré group is determined by an integer generator $g \geq 2$. All the Lorentz boosts in $\mathbf{L}$ must be in the same direction. It is either in the $x$-direction or in the $y$-direction. Without loss of generality, we suppose it to be in the $x$-direction. The temporal primitive vector of $\mathbf{Y}$ is then $r_{0}^{(e)}=\left(\sqrt{g^{2}-4} a_{1} /(2 c), 0,0\right)^{T}$ for even $g$, or $r_{0}^{(o)}=\left(\sqrt{g^{2}-4} a_{1} /(2 c), a_{1} / 2,0\right)^{T}$ for odd $g$. An arbitrary vector of $\mathbf{Y}$ can be expressed as

$$
r_{n_{0} n_{1} n_{2}}=n_{0} r_{0}+n_{1} r_{1}+n_{2} r_{2},
$$

where $n_{0}, n_{1}$ and $n_{2}$ are integers. Fig. 1 shows a unit cell of $\mathbf{Y}$. $\mathbf{Y}$ in $1+2$ dimensions is indeed an extension of the 1+1-dimensional spacetime lattice along a perpendicular direction. $\mathbf{Y}$ is an orthorhombic lattice for even $g$ or a base-centered orthorhombic lattice for odd $g$. 

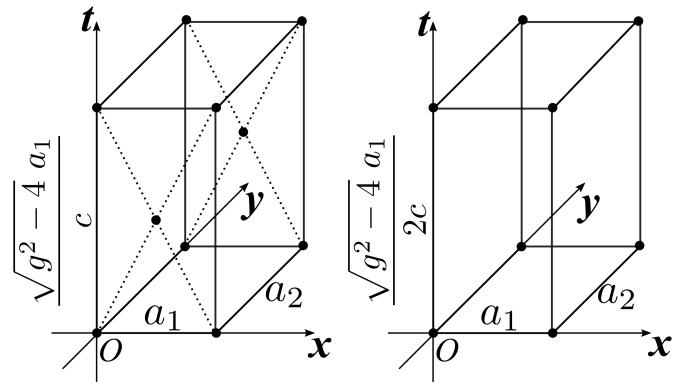

FIG. 1. A unit cell of $\mathbf{Y}$ for $2 \mathrm{D}$ rectangular lattice. The left panel is for odd $g$, while the right panel is for even $g$.

The Lorentz boost in $\mathbf{L}$ can be expressed as

$$
B_{v_{j}}=\left(\begin{array}{ccc}
\frac{1}{\sqrt{1-v_{j}^{2} / c^{2}}} & \frac{-v_{j} / c^{2}}{\sqrt{1-v_{j}^{2} / c^{2}}} & 0 \\
\frac{-v_{j}}{\sqrt{1-v_{j}^{2} / c^{2}}} & \frac{1}{\sqrt{1-v_{j}^{2} / c^{2}}} & 0 \\
0 & 0 & 1
\end{array}\right)
$$

where $v_{j}=\operatorname{sign}(j) \sqrt{1-4 / m_{j}^{2}(g)} c$ takes the same value as in $1+1$ dimensions. $B_{v_{j}}$ acting on a vector keeps its $y$-component invariant. The transformation is purely within the $t-x$ plane.

We use $\mathbf{R}$ to denote the point group of a Bravais lattice. For the rectangular lattice, $\mathbf{R}$ contains a rotation of angle $\pi$ in the $x-y$ plane, a reflection across the $x$-axis and a reflection across the $y$-axis. The discrete Lorentz group $\mathbf{L}$ can then be expressed as

$$
\mathbf{L}=\left\{R B_{v_{j}} \mid R \in \mathbf{R} \text { and } j=0, \pm 1, \pm 2, \cdots\right\} .
$$

The element of $\mathbf{L}$ is the product of a spatial operation and a Lorentz boost. In the case $g=2, B_{v_{j}}$ is the identity matrix and $\mathbf{L}$ reduces to $\mathbf{R}$, that is the Lorentz symmetry is absent. For $g>2$, $\mathbf{L}$ gives a discrete Lorentz symmetry in the $x$-direction, but no Lorentz symmetry in the $y$ direction.

\section{THREE DIMENSIONAL BRAVAIS LATTICES}

In three spatial dimensions, there are 14 Bravais lattices. There exists symmorphic Poincaré symmetry on the monoclinic, orthorhombic, base-centered orthorhombic, tetragonal and hexagonal lattices (see table \). The derivation is given in appendix D.

We denote a vector in 1+3-dimensional spacetime as $(t, x, y, z)^{T}$. Again, $\mathcal{P}=\mathbf{L} \times \mathbf{Y}$ denotes the symmorphic Poincaré group. The Lorentz boosts in $\mathbf{L}$ are supposed to be in the $x$-direction without loss of generality. Similar to $1+2$-dimensional case, the Lorentz boost is expressed

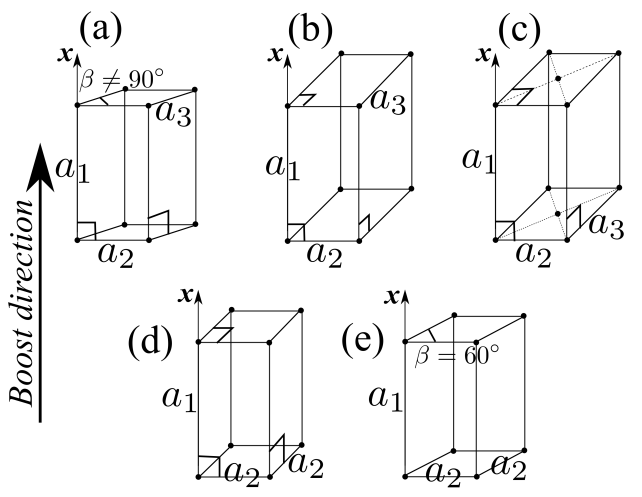

FIG. 2. The boost direction on the (a) monoclinic, (b) orthorhombic, (c) base-centered orthorhombic, (d) tetragonal and (e) hexagonal lattices. $a_{1}, a_{2}$ and $a_{3}$ denote the lattice constants. And we use $\beta$ to denote the angle between two primitive vectors in the $y$ - $z$ plane when it is not $90^{\circ}$.

as

$$
B_{v_{j}}=\left(\begin{array}{cccc}
\frac{1}{\sqrt{1-v_{j}^{2} / c^{2}}} & \frac{-v_{j} / c^{2}}{\sqrt{1-v_{j}^{2} / c^{2}}} & 0 & 0 \\
\frac{-v_{j}}{\sqrt{1-v_{j}^{2} / c^{2}}} & \frac{1}{\sqrt{1-v_{j}^{2} / c^{2}}} & 0 & 0 \\
0 & 0 & 1 & 0 \\
0 & 0 & 0 & 1
\end{array}\right)
$$

where $v_{j}$ takes the same value as in $1+1$ or $1+2$ dimensions. And $\mathbf{L}$ is again $\left\{R B_{v_{j}} \mid R \in \mathbf{R}\right.$ and $\left.j=0, \pm 1, \pm 2, \cdots\right\}$, where $\mathbf{R}$ is the point group of the corresponding Bravais lattice. There is no Lorentz symmetry in the $y$ - $z$ plane.

$\mathbf{Y}$ in $1+3$ dimensions has four primitive vectors. We use $r_{1}, r_{2}$ and $r_{3}$ to denote the three primitive vectors of the Bravais lattice. If $r_{1}$ is chosen to be $\left(0, a_{1}, 0,0\right)^{T}$, the temporal primitive vector is then $r_{0}^{(e)}=\left(\sqrt{g^{2}-4} a_{1} /(2 c), 0,0,0\right)^{T}$ for even $g$, or $r_{0}^{(o)}=$ $\left(\sqrt{g^{2}-4} a_{1} /(2 c), a_{1} / 2,0,0\right)^{T}$ for odd $g$. A vector of $\mathbf{Y}$ can be expressed as

$$
r_{n_{0} n_{1} n_{2} n_{3}}=n_{0} r_{0}+n_{1} r_{1}+n_{2} r_{2}+n_{3} r_{3},
$$

where $n_{0}, n_{1}, n_{2}$ and $n_{3}$ are integers.

Note that $r_{1}$ cannot be chosen arbitrarily. It is required that $r_{2}$ and $r_{3}$ be both perpendicular to $r_{1}$. Fig. 2 displays the direction of $r_{1}$ on different Bravais lattices. The monoclinic, orthorhombic, base-centered orthorhombic, tetragonal and hexagonal lattices can be viewed as the five 2D Bravais lattices extended in the perpendicular direction, respectively. And this perpendicular direction must be chosen to the direction of $r_{1}$. Especially, in the tetragonal lattice, $r_{1}$ must lie in the vertical direction in which the lattice constant is different from those in the other two directions.

Again, the discrete Poincaré group $\mathcal{P}$ is uniquely determined by the integer generator $g \geq 2$. In the case $g=2$, 
we obtain $B_{v_{j}} \equiv 1$ and $\mathbf{L} \equiv \mathbf{B}$. And $r_{0}=0$ indicates a continuous TTS. The symmetry group reduces to the space group of Bravais lattices.

In the case $g>2, v_{j}(g)=\operatorname{sign}(j) \sqrt{1-4 / m_{j}^{2}} c$ is nonzero for $j \neq 0$. When one reference frame is moving at $v_{j}$ relative to the other, the Bravais lattice looks the same for them. And the temporal primitive vector is now nonzero, indicating that TTS is broken into a discrete one.

\section{DISCUSSION}

We have enumerated the symmorphic Poincaré groups on 2D and 3D Bravais lattices. An interesting question is whether the symmorphic Poincaré symmetry does exist in real crystals. According to our results, it is possible to find such a symmetry only in crystals based on the monoclinic, orthorhombic, base-centered orthorhombic, tetragonal or hexagonal lattices. For examples, graphite and rutile are based on the hexagonal and tetragonal Bravais lattices, respectively.

If a crystal has symmorphic Poincaré symmetry, it keeps invariant under a coordinate transformation of $\mathcal{P}=\mathbf{L} \times \mathbf{Y}$, where $\mathbf{L}$ and $\mathbf{Y}$ are determined by the generator $g$. Each crystal has a unique $g$. To be more precise, when we describe the motion of electrons (or phonons) in this crystal, the Lagrangian density or equation of motion need to keep invariant under $\mathcal{P}$, which imposes a strong constraint on the possible form of them. Here, the Lagrangian (or equation of motion) is an effective one, only for the particles that we are interested in. The full Lagrangian for the electrons, nuclei and their interactions has of course the continuous Poincaré symmetry. One can think that the effective one is derived from the full one by using some mean-field theory. Ref. [12] showed how to construct such an effective Lagrangian from the symmetry principle. One starts from a Lagrangian with continuous Poincaré symmetry and then replaces the constants (coupling or mass) by a function $f(r)$ that has the symmetry $\mathcal{P}$. Especially, $f(r)$ is invariant under a translation of vector $r_{n_{0} n_{1} n_{2} n_{3}}$, where $r_{n_{0} n_{1} n_{2} n_{3}}$ is given by Eq. (8). As $n_{0}=0$, this means that $f(r)$ is a periodic function of space. As $n_{0} \neq 0$, we find that $f(r)$ is also a periodic function of time:

$$
f(t, x, y, z)=f(t+T, x, y, z) .
$$

It is easy to see $T=\sqrt{g^{2}-4} a_{1} /(2 c)$ for even $g$ or $T=\sqrt{g^{2}-4} a_{1} / c$ for odd $g$. Here $a_{1}$ is the lattice constant. Since the coupling $f$ in the equation of motion is a periodic function, we expect the solutions to be also periodic functions with the same period. Therefore, the local properties of the crystal should change periodically in the spacetime.

If the generator of a crystal is $g=2$, we obtain $T=0$. In this case, $f$ is independent of time, so are the local properties. This is what we usually think of. But there exist the other possibilities. If the generator of a crystal is $g>2$, its local properties change periodically with time, even in the absence of external driving. This is the exclusive feature of symmorphic Poincaré symmetry. An experiment searching for the time periodicity in crystals will then clarify whether there exists symmorphic Poincaré symmetry or not. Let us take graphite as an example. Its lattice constant is $a_{1} \approx 6.7 \times 10^{-10} \mathrm{~m}$. Its time period is then $T=\sqrt{g^{2}-4} \times 1.1 \times 10^{-18} s$ for even $g$ or $T=\sqrt{g^{2}-4} \times 2.2 \times 10^{-18} s$ for odd $g$. The time periods of typical crystals are very small. This may explain why the symmorphic Poincaré symmetry has not been observed up to now. An alternative way of observing the time periodicity would be by using the Floquet effect. The function $f(r)$ in the equation of motion can be treated as a periodically-driving potential. In the presence of it, we expect the system to absorb radiation of the frequency $1 / T$. Therefore, a peak at this frequency in the absorption spectrum would also support the existence of discrete Poincaré symmetry. We use again graphite as an example. The frequency is $\left(g^{2}-4\right)^{-1 / 2} \times 9.1 \times 10^{17} \mathrm{~Hz}$ for even $g$ or $\left(g^{2}-4\right)^{-1 / 2} \times 4.5 \times 10^{17} \mathrm{~Hz}$ for odd $g$. It is in the frequency range of $\mathrm{X}$-rays.

This paper focuses on the classification of discrete Poincaré symmetry. In future, we expect to study the effective field theories with this symmetry and how to obtain them from a fundamental theory with continuous Poincaré symmetry.

\section{ACKNOWLEDGEMENT}

This work is supported by NSFC under Grant No. 11774315. P. Wang is also supported by the Junior Associates program of the Abdus Salam International Center for Theoretical Physics.

Xiuwen Li, Jiaxue Chai and Huixian Zhu contribute equally to this paper.

\section{Appendix A: Proof of $\mathrm{L} \times \mathrm{Y}$ being a symmorphic Poincaré group}

Suppose $\mathbf{L}=\{L\}$ is a group of Lorentz transformations and $\mathbf{Y}=\{r\}$ is a group of translations. We express the set $\mathbf{L} \times \mathbf{Y}$ as $\mathcal{P}=\{\Lambda(L, r) \mid L \in \mathbf{L}, r \in \mathbf{Y}\}$. According to definition, $\mathcal{P}$ is a symmorphic Poincaré group if and only if $\mathcal{P}$ is a group under the multiplication rule (11). Therefore, our destination is to prove that $\mathcal{P}$ is a group under the rule (11) if and only if $\mathbf{Y}$ is invariant under $\mathbf{L}$.

First, we prove that $\mathcal{P}$ is a group if $\mathbf{Y}$ is invariant under $\mathbf{L}$. The associativity of the multiplication rule (1) is obvious. If $\Lambda(L, r)$ and $\Lambda\left(L^{\prime}, r^{\prime}\right)$ are two elements of $\mathcal{P}$, we have $L, L^{\prime} \in \mathbf{L}$ and $r, r^{\prime} \in \mathbf{Y}$ according to definition. By using Eq. (1), we obtain $\Lambda(L, r) \Lambda\left(L^{\prime}, r^{\prime}\right)=$ $\Lambda\left(L L^{\prime}, L r^{\prime}+r\right)$. Since $\mathbf{L}$ is a group, $L L^{\prime} \in \mathbf{L}$ is obvious. And because $\mathbf{Y}$ is invariant under $\mathbf{L}$, we obtain 
$L r^{\prime} \in \mathbf{Y}$ and then $L r^{\prime}+r \in \mathbf{Y}$ ( $\mathbf{Y}$ is a group). Therefore, $\Lambda\left(L L^{\prime}, L r^{\prime}+r\right)$ must be an element of $\mathcal{P}$. The closure of $\mathcal{P}$ is proved. $\mathbf{Y}$ and $\mathbf{L}$ are groups, so that they contain identity elements. The identity element of $\mathbf{Y}$ is $r=0$ (no translation), and the identity element of $\mathbf{L}$ is $L=1$ (no Lorentz rotation). By using Eq. (11), it is easy to see $\Lambda(1,0) \Lambda(L, r)=\Lambda(L, r) \Lambda(1,0)=\Lambda(L, r)$ for arbitrary $L$ and $r$. Therefore, $\Lambda(1,0)$ is the identity element of $\mathcal{P}$. The existence of identity element is proved. If $\Lambda(L, r)$ is an element of $\mathcal{P}$, we have $L \in \mathbf{L}$ and then $L^{-1} \in \mathbf{L}$, and $r \in \mathbf{Y}$. Since $\mathbf{Y}$ is invariant under $\mathbf{L}$, we obtain $L^{-1} r \in \mathbf{Y}$ and then $-L^{-1} r \in \mathbf{Y}$. Therefore, $\Lambda\left(L^{-1},-L^{-1} r\right)$ is also an element of $\mathcal{P}$ according to definition. And it is easy to see that $\Lambda\left(L^{-1},-L^{-1} r\right)$ is the inverse of $\Lambda(L, r)$. The existence of inverse element is proved. As above, if $\mathbf{Y}$ is invariant under $\mathbf{L}, \mathcal{P}$ must be a group.

Second, we prove that $\mathcal{P}$ is not a group if $\mathbf{Y}$ is not invariant under $\mathbf{L}$. If $\mathbf{Y}$ is not invariant under $\mathbf{L}$, there exist $L \in \mathbf{L}$ and $r \in \mathbf{Y}$ so that $L r \notin \mathbf{Y}$. Because $\mathbf{L}$ and $\mathbf{Y}$ are groups and the set $\mathcal{P}$ is the direct product of $\mathbf{L}$ and $\mathbf{Y}$, we know that $\Lambda(L, 0), \Lambda(1, r)$ and $\Lambda\left(L^{-1}, 0\right)$ are the elements of $\mathcal{P}$. Their product is $\Lambda(L, 0) \Lambda(1, r) \Lambda\left(L^{-1}, 0\right)=$ $\Lambda(1, L r)$. But $\Lambda(1, L r)$ is not an element of $\mathcal{P}$. Therefore, $\mathcal{P}$ is not closed under the rule (10). $\mathcal{P}$ is not a group.

As above, $\mathcal{P}$ is a group if and only if $\mathbf{Y}$ is invariant under $\mathbf{L}$.

\section{Appendix B: Proof of $\vec{v}$ being in the lattice direction}

Suppose $\mathbf{Y}$ is a spacetime lattice obtained by extending a Bravais lattice in the time direction. $B_{\vec{v}}$ is a Lorentz boost, and it is an element of $\mathbf{L}$. In this appendix, we prove that $\mathbf{Y}$ is invariant under $\mathbf{L}$ only if $\vec{v}$ is in the lattice direction.

In $1+d$ dimensions, we can express the spacetime coordinates as $\left(t, x_{1}, \cdots, x_{d}\right)^{T}$. Without loss of generality, we suppose that $\vec{v}$ is confined to the $x_{1}$-direction. The matrix $B_{\vec{v}}$ then looks like

$$
B_{\vec{v}}=\left(\begin{array}{cccc}
\frac{1}{\sqrt{1-v^{2} / c^{2}}} & \frac{-v / c^{2}}{\sqrt{1-v^{2} / c^{2}}} & 0 & \ldots \\
\frac{-v}{\sqrt{1-v^{2} / c^{2}}} & \frac{1}{\sqrt{1-v^{2} / c^{2}}} & 0 & \ldots \\
0 & 0 & 1 & \ldots \\
\cdots & \ldots & \ldots & \ldots
\end{array}\right) .
$$

$B_{\vec{v}}$ is a symmetric matrix if we use the unit $c=1$. The diagonal elements are all 1 and the off-diagonal elements are all zero except for the first two lines and columns. Suppose $r \in \mathbf{Y}$ is a Bravais lattice vector, hence its temporal component is zero. We choose $r=\left(0, x_{1}, \cdots, x_{d}\right)^{T}$ with $x_{1} \neq 0$ without loss of generality. Indeed, there always exist lattice vectors with nonzero component in the $x_{1}$-direction, otherwise, it is not a Bravais lattice.

If $\mathbf{Y}$ is invariant under $B_{\vec{v}}, B_{\vec{v}} r$ must be an element of $\mathbf{Y}$. And $B_{\vec{v}} \in \mathbf{L}$ infers $B_{-\vec{v}} \in \mathbf{L}$. Therefore, $B_{-\vec{v}} r$ is an element of $\mathbf{Y}$, hence $r^{\prime}=B_{\vec{v}} r+B_{-\vec{v}} r-2 r$ is an element of $\mathbf{Y}$. Here we have used the properties of $\mathbf{Y}$ being a group. $r^{\prime}$ reads $\left(0, k x_{1}, 0, \cdots\right)^{T}$ with $k=\frac{2}{\sqrt{1-v^{2} / c^{2}}}-2$. We have $k>0$ for $v \neq 0$, and then $r^{\prime} \neq 0$. According to the definition of $\mathbf{Y}, r^{\prime}$ is a Bravais lattice vector since its temporal component is zero. And $r^{\prime}$ lies in the $x_{1}$-axis. This means that the $x_{1}$-direction is a lattice direction. We then proved that $\vec{v}$ is in the lattice direction.

\section{Appendix C: (1+2)-dimensional symmorphic Poincaré groups}

In this section, we construct the symmorphic Poincaré groups in $1+2$ dimensions. We use $(t, x, y)^{T}$ to denote the coordinates in $1+2$ dimensions. Suppose $\mathcal{P}=\mathbf{L} \times \mathbf{Y}$ is a symmorphic Poincaré group. $\mathbf{Y}$ is invariant under L. For nontrivial $\mathcal{P}, \mathbf{L}$ contains at least one Lorentz boost $B_{\vec{v}}$. And $\mathbf{Y}$ is invariant under $B_{\vec{v}}$. As proved in appendix B $\vec{v}$ must be in the lattice direction. Without loss of generality, we suppose that $\vec{v}$ is in the $x$-direction and the lattice constant in this direction is $a_{1}$. Therefore, $r_{1}=\left(0, a_{1}, 0\right)^{T}$ is a primitive vector of the Bravais lattice, and a primitive vector of $\mathbf{Y}$ as well.

$B_{\vec{v}}$ can be expressed as

$$
B_{\vec{v}}=\left(\begin{array}{ccc}
\frac{1}{\sqrt{1-v^{2} / c^{2}}} & \frac{-v / c^{2}}{\sqrt{1-v^{2} / c^{2}}} & 0 \\
\frac{-v}{\sqrt{1-v^{2} / c^{2}}} & \frac{1}{\sqrt{1-v^{2} / c^{2}}} & 0 \\
0 & 0 & 1
\end{array}\right),
$$

which is a symmetric matrix in the unit $c=1 . B_{\vec{v}}$ rotates $r_{1}$ into the $t-x$ plane. The sublattice of $\mathbf{Y}$ in the $t$ - $x$ plane is a $(1+1)$-dimensional spacetime lattice, which is invariant under $B_{\vec{v}}$. Obviously, this sublattice and $B_{\vec{v}}$ are the elements of a $(1+1)$-dimensional discrete Poincaré group. As reviewed in Sec. III such a group is determined by an integer generator $g \geq 2$. For an even $g$, the primitive vectors of the sublattice are $r_{1}$ and $r_{0}^{(e)}=$ $\left(\sqrt{g^{2}-4} a_{1} /(2 c), 0,0\right)^{T}$. For an odd $g$, the primitive vectors are $r_{1}$ and $r_{0}^{(o)}=\left(\sqrt{g^{2}-4} a_{1} /(2 c), a_{1} / 2,0\right)^{T}$. And the velocity in the Lorentz boost can only take the values

$$
v_{j}=\operatorname{sign}(j) \sqrt{1-4 / m_{j}^{2}(g)} c
$$

with $j=0, \pm 1, \pm 2, \cdots$.

The lattice $\mathbf{Y}$ has three primitive vectors in $1+2$ dimensions. Among them, $r_{0}$ and $r_{1}$ lie in the $t-x$ plane. While the Bravais lattice has two primitive vectors in the spatial dimensions, namely $r_{1}$ and $r_{2}$. Obviously, $r_{0}, r_{1}$ and $r_{2}$ must be the three primitive vectors of $\mathbf{Y}$.

Without loss of generality, we express $r_{2}$ as $\left(0, r_{2 x}, r_{2 y}\right)^{T}$. An arbitrary vector of $\mathbf{Y}$ (dubbed a lattice vector) can be expressed as $n_{0} r_{0}+n_{1} r_{1}+n_{2} r_{2}$ with $n_{0}, n_{1}$ and $n_{2}$ being integers. $\mathbf{Y}$ is invariant under $B_{\vec{v}}$ if and only if $B_{\vec{v}} r_{0}, B_{\vec{v}} r_{1}$ and $B_{\vec{v}} r_{2}$ are lattice vectors. $B_{\vec{v}} r_{0}$ and $B_{\vec{v}} r_{1}$ are obviously lattice vectors, since $r_{0}$ and $r_{1}$ are 
the primitive vectors of the $(1+1)$-dimensional sublattice which is invariant under $B_{\vec{v}}$.

Let us study the condition of $B_{\vec{v}} r_{2}$ being a lattice vector. The value of $\vec{v}$ is given by Eq. (C2). And we already know from Ref. [12] that $B_{\vec{v}_{j}}=\left(B_{\vec{v}_{1}}\right)^{j}$, where the superscript denotes the exponent. Therefore, $B_{\vec{v}_{j}} r_{2}$ is a lattice vector if and only if $B_{\vec{v}_{1}} r_{2}$ and $B_{-\vec{v}_{1}} r_{2}$ are lattice vectors. Note $\left(B_{\vec{v}_{1}}\right)^{-1}=B_{-\vec{v}_{1}}$. And the elementary Lorentz boost is

$$
B_{ \pm \vec{v}_{1}}=\left(\begin{array}{ccc}
\frac{g}{2} & \mp \frac{1}{c} \sqrt{\frac{g^{2}}{4}-1} & 0 \\
\mp \sqrt{\frac{g^{2}}{4}-1} c & \frac{g}{2} & 0 \\
0 & 0 & 1
\end{array}\right) .
$$

Now we obtain two equations:

$$
\begin{aligned}
B_{\vec{v}_{1}} r_{2} & =n_{0} r_{0}+n_{1} r_{1}+n_{2} r_{2} \\
B_{-\vec{v}_{1}} r_{2} & =\bar{n}_{0} r_{0}+\bar{n}_{1} r_{1}+\bar{n}_{2} r_{2} .
\end{aligned}
$$

We need to find $r_{2 x}$ and $r_{2 y}$ so that $n_{0}, n_{1}, n_{2}, \bar{n}_{0}, \bar{n}_{1}$, $\bar{n}_{2}$ are all integers. It is easy to see that $r_{2 x}$ must be an integer times of $a_{1}$ but $r_{2 y}$ can be arbitrary real number. Without loss of generality, we choose $r_{2 x}=0$ and rename $r_{2 y}$ as $a_{2}$. The second primitive vector of the Bravais lattice is then $r_{2}=\left(0,0, a_{2}\right)^{T}$.

In one word, if $\mathcal{P}=\mathbf{L} \times \mathbf{Y}$ is a symmorphic Poincaré group and $\mathbf{L}$ contains at least one Lorentz boost, two primitive vectors of the Bravais lattice must be perpendicular to each other and the velocity of the boost is in the direction of one primitive vector.

With this in mind, we rule out the possibility of oblique, centered rectangular or hexagonal lattices owning nontrivial symmorphic Poincaré symmetry. Further analysis rules out the possibility of the square lattice. This can be proved by contradiction. We suppose that a square lattice has the primitive vectors $r_{1}=(0, a, 0)^{T}$ and $r_{2}=(0,0, a)^{T}$. Its spacetime lattice $\mathbf{Y}$ is invariant under the Lorentz boost $B_{\vec{v}}$ with $\vec{v}$ lying in the $x$ direction. $B_{\vec{v}}$ is an element of $\mathbf{L}$. Do not forget that $\mathbf{L}$ contains the point group of the square lattice. In particular, a rotation of angle $\pi / 2$ in the $x-y$ plane is an element of $\mathbf{L}$, which can be expressed as

$$
R_{\pi / 2}=\left(\begin{array}{ccc}
1 & 0 & 0 \\
0 & 0 & -1 \\
0 & 1 & 0
\end{array}\right)
$$

$B^{\prime}=R_{\pi / 2} B_{\vec{v}} R_{\pi / 2}^{-1}$ is then a Lorentz boost in the $y$ direction. And $\left(B_{\vec{v}} B^{\prime} B^{\prime} B_{\vec{v}}\right) \in \mathbf{L}$ is a Lorentz boost of velocity $\vec{v}^{\prime}$. $\vec{v}^{\prime}$ makes an angle $0<\theta<\pi / 2$ with the positive $x$-axis. As proved in above, $\vec{v}^{\prime}$ must be in a lattice direction of the square lattice, since $\mathbf{Y}$ is also invariant under $\left(B_{\vec{v}} B^{\prime} B^{\prime} B_{\vec{v}}\right)$. And if we use $r_{1}^{\prime}$ to denote the primitive vector of the square lattice in the $\vec{v}^{\prime}$-direction, the other primitive vector $r_{2}^{\prime}$ has to be perpendicular to $r_{1}^{\prime}$. But this is impossible on a square lattice. Therefore, a square lattice cannot have nontrivial symmorphic Poincaré symmetry.

In two spatial dimensions, there exist nontrivial symmorphic Poincaré symmetry only on the rectangular lattice. If we choose two perpendicular primitive vectors $r_{1}=\left(0, a_{1}, 0\right)^{T}$ and $r_{2}=\left(0,0, a_{2}\right)^{T}$ with $a_{1} \neq a_{2}$, the Lorentz boost must be in the direction of $r_{1}$ or $r_{2}$.

\section{Appendix D: (1+3)-dimensional symmorphic Poincaré groups}

In this section, we construct the symmorphic Poincaré groups in $1+3$ dimensions. We use $(t, x, y, z)^{T}$ to denote the coordinates in $1+3$ dimensions. Again, we suppose $\mathcal{P}=\mathbf{L} \times \mathbf{Y}$ is a symmorphic Poincaré group. And $\mathbf{L}$ contains at least one Lorentz boost $B_{\vec{v}}$. $\vec{v}$ is in the $x$ direction and the lattice constant in this direction is $a_{1}$. $r_{1}=\left(0, a_{1}, 0,0\right)^{T}$ is a primitive vector of the Bravais lattice. The other two primitive vectors are supposed to be $r_{2}=\left(0, r_{2 x}, r_{2 y}, r_{2 z}\right)^{T}$ and $r_{3}=\left(0, r_{3 x}, r_{3 y}, r_{3 z}\right)^{T}$.

Similarly, we can prove that $r_{2 x}=0$ and $r_{3 x}=0 . r_{2}$ and $r_{3}$ must be perpendicular to $r_{1}$. And the temporal primitive vector of $\mathbf{Y}$ is $r_{0}^{(e)}=\left(\sqrt{g^{2}-4} a_{1} /(2 c), 0,0,0\right)^{T}$ for even $g$ or $r_{0}^{(o)}=\left(\sqrt{g^{2}-4} a_{1} /(2 c), a_{1} / 2,0,0\right)^{T}$ for odd $g$.

In three-dimensional Bravais lattices, the boost must be in the direction of one primitive vector and the other two primitive vectors are in the perpendicular direction. There are 14 Bravais lattices. Only in the monoclinic, orthorhombic, base-centered orthorhombic, tetragonal, hexagonal or cubic lattices, there exist two primitive vectors that are both perpendicular to the third one. But similar to the square lattice in two dimensions, the cubic lattice cannot have nontrivial symmorphic Poincaré symmetry.

Notice that the monoclinic, orthorhombic, basecentered orthorhombic, tetragonal and hexagonal lattices can be created by extending five $2 \mathrm{D}$ Bravais lattices in the perpendicular direction. And this direction is indeed the direction of the Lorentz boost.

\footnotetext{
* wangpei@zjnu.cn

1 A. Shapere and F. Wilczek, Physical Review Letters 109, 160402 (2012).

${ }^{2}$ F. Wilczek, Physical Review Letters 109, 160401 (2012).
}

${ }^{3}$ P. Bruno, Physical Review Letters 111, 070402 (2013).

${ }^{4}$ H. Watanabe and M. Oshikawa, Physical Review Letters 114, 251603 (2015).

${ }^{5}$ D. V. Else, B. Bauer, and C. Nayak, Physical Review Let- 
ters 117, 090402 (2016).

${ }^{6}$ K. Sacha, Physical Review A 91, 033617 (2015).

7 V. Khemani, A. Lazarides, R. Moessner, and S. L. Sondhi, Physical Review Letters 116, 250401 (2016).

8 J. Zhang, P. W. Hess, A. Kyprianidis, P. Becker, A. Lee, J. Smith, G. Pagano, I.-D. Potirniche, A. C. Potter, A. Vishwanath, et al., Nature 543, 217 (2017).

9 S. Choi, J. Choi, R. Landig, G. Kucsko, H. Zhou, J. Isoya, F. Jelezko, S. Onoda, H. Sumiya, V. Khemani, et al., Nature 543, 221 (2017).

10 M. P. Marder, Condensed Matter Physics (John Wiley \& Sons, New Jersey, 2010).

11 S. Weinberg, The Quantum Theory of Fields (Cambridge University Press, Cambridge, 2005).
12 P. Wang, New J. Phys. 20, 023042 (2018).

13 L. Bombelli, J. Lee, D. Meyer, and D. S. Rafael, Physical Review Letters 59, 521 (1987).

14 R. D. Sorkin, arXiv:0309009 (2003).

15 H. Yamamoto, Nuclear Physics B (Proc. Suppl.) 6, 154 (1989).

16 E. R. Livine and D. Oriti, Journal of High Energy Physics 06, 050 (2004)

17 M. A. Rieffel, Mem. Amer. Math. Soc. 168, 1 (2004).

18 M. A. Rieffel, Mem. Amer. Math. Soc. 168, 67 (2004).

19 Note1, there exist possibilities that $\mathbf{L}$ contains no Lorentz boost but only composite Lorentz transformations ( $L=$ $R B)$, which are not discussed in this paper. 